\title{
Bounded variation and invariant measures
}

by

MAREK RYCHLIK (Warszawa)

Ahstract. In this paper we study the existence and properties of invariant measures absolutely continuous with respect to the Lebesgue measure for piocewise continuous expanding maps of an interval with finitely or infinitely many pieces of monotonicity. The technique of bounded variation is used. The Bernoulli property and some limit thoorems are proved for resulting dynamical systems.

Introduction. Wo study the existence and ergodic properties of invariant measures for various maps. The Bernoulli property and the central limit theorem are also discussed. Our formalism is similar to that of Hofbauer and Keller [1]. Our paper allows us to extend the results of [1], so that the maps with infinitely many pieces of monotonicity are included in the general theory, in particular, the maps considered by Walters [2].

The way we have chosen is different from that of Hofbauer and Keller. Their method relies on the theorem of Tuleea-Romanesen and Marinescu. Instead of that we apply the uniform ergodic theory. Of course, slightly modifying our method we can follow [1]. On the other hand, key estimations in their paper cannot be used in our case. Besides, we present simplified proofs of many facts, in particular the Bernoulli property and limit theorems. We have also explained the cyclic structure of the Perron-Frobenius operation.

Our referene list is not eomplete. The render can find a lot of literatiure in [1].

\$1. Iset $x$ bo a totidly ordexed ordex-eompleto seti. Open intervals constitutio a base of a compast topology in $X$, making $X$ into a topologieal space. If $X$ is separable, then $X$ is homeomorphie with a closed subset of an interval. By $Q B$ we denote the o-algebra of all Borel subsets of $X$. Wo fix an regular, Jorel probabilistice measure $m$ on $X$.

Thee functional spaces will appear below. Two of them are well known: $L_{\infty}(m)$ and $I_{1}(m)$. We often write $I_{\infty}$ and $L_{1}$. Norms in these spaces axe denoted by $\|\cdot\|_{\infty}$ and $\|\cdot\|_{1}$, respectively. Given $f: X \rightarrow R$ wo 
define variation of $f$ on a subset $O \subset X$ :

$$
\operatorname{Var}_{C} f=\sup \left\{\sum_{i=1}^{p}\left|f\left(x_{i}\right)-f\left(x_{i-1}\right)\right|\right\}
$$

where the supremum is over all sequences $\left(x_{0}, \ldots, x_{p}\right), x_{0}, \ldots, x_{p} \in C$ and $x_{0} \leqslant x_{1} \leqslant \ldots \leqslant x_{p}$. In place of $\operatorname{Var}_{X} f$ we write Varf.

$$
\text { Let }
$$

$$
\mathrm{BV}=\left\{f \in I_{\infty}: f \text { has a version of bounded variation }\right\} \text {. }
$$

$\mathrm{BV}$ is a Banach space with the norm

$$
\|f\|=\max \left(\|f\|_{1}, \inf \{\operatorname{Var} \tilde{f}: \tilde{f} \text { is a version of } f\}\right) .
$$

Remark 1. Every $f \in \mathrm{BV}$ has a version $\tilde{f}$ with minimal variation. This holds iff for every $x_{0} \in X$

$$
\tilde{f}\left(x_{0}\right) \in\left[\lim _{x \rightarrow x_{0}(-)} \tilde{f}, \lim _{x \rightarrow x_{0}(+)} \tilde{f}\right] .
$$

One-sided limits always exist for $\tilde{f}$. If we do not talk about versions of of $f$ explicitly, we assume that we have chosen a version satisfying (4). We notice that Varf does not depend on the choice of $\tilde{f}$ with this property.

Let $T: U \rightarrow X$ be a continuous map, $U \subset X$ is open and dense, and $m(U)=1$. Let $S=X \backslash U$. We assume that:

There exists a countable family $\beta$ of closed intervals with disjoint interiors such that $\bigcup \beta \supset U$ and for any $B \in \beta$ the set $B \cap S$ consists exactly of the endpoints of $B$.

For any $B \in \beta,\left.T\right|_{B \cap U}$ admits an extension to a homeomorphism of $B$ with some interval in $X$.

A function $g: X \rightarrow R_{+}$is given, $\|g\|_{\infty}<1, \operatorname{Var} g<+\infty,\left.g\right|_{S}=0$ and the operator $P: L_{1} \rightarrow L_{1}$ defined by

$$
P f(x)=\sum_{y \in T^{\prime}=1} g(y) f(y)
$$

preserves $m$, which means $m(P f)=m(f)$ for anch $f \in L_{1}$.

All subsets of $X$ that ocetr below are measurable.

Remark 2. $P$ is the Perron-Frobonius operator for $T$.

The proof of this fact will be divided into steps (a), (b) and (c).

(a) $T$ is non-singular.

Proof. For any $f, h \in I_{1}$ such that $f \cdot h \in I_{1}, I(f \circ T \cdot h) \cdots f \cdot I \cdot h$. We put $f=\chi_{A}, A \subset X$, and $h=\chi_{B}, B \in \beta$. So

$$
\begin{aligned}
m\left(T^{-1} A \cap B\right) & =m\left(\chi_{A} \circ T_{\chi_{B 3}}\right)=m\left(P\left(\chi_{A 1} \circ T \cdot \chi_{B B}\right)\right)=m\left(\chi_{A L} \cdot P \chi_{X_{1}}\right) \\
& \leqslant m(A \cap T B)\|g\|_{\infty}
\end{aligned}
$$

sinee $\left.P X_{X_{B 3}}\right|_{X \backslash T H 3}=0$ and $\left\|P_{\chi_{I B}}\right\|_{\infty} \leqslant\|g\|_{\infty}$. Thus, $m(A)=0$ implies

$$
m\left(T^{-1} A\right) \leqslant \sum_{B \in \beta} m\left(T^{-1} A \cap B\right)=
$$

(b) $g \neq 0$ a.e.

Proof. $m\left(g^{-1}(0)\right)=m\left(P_{\chi_{0}-1(0)}\right)=0$, because $P\left(\chi_{g}-1(0)\right)=0$.

(c) Put $J=1 / g$ where it exists. Then $J$ is the jacobian of $T$.

Proof. For every $B \in \beta$ and $A \subset X$ :

$$
\begin{aligned}
m(T B \cap A) & =m\left(\chi_{A} \cdot P\left(\chi_{A B} \cdot J\right)\right)=m\left(P\left(\chi_{A} \circ T \cdot \chi_{I B} \cdot J\right)\right)=m\left(\chi_{A} \circ T \cdot \chi_{I S} \cdot J\right) \\
& =\int_{T \rightarrow 1} J d m
\end{aligned}
$$

sines $T^{\prime}\left(\chi_{I} \cdot J\right)=\chi_{T / B}$ a.c.

We are going to check that iterations of $T$ satisfy our conditions. For our purposes, a partition will mean a countable family of closed intervals such that each two of them can have only an endpoint in common and exhausting $X$ up to a set of measure 0 . So $\beta$ is a partition.

Puti $S_{N}=\bigcup_{l=0 a 0}^{N-1} T^{-k}(S)$ and $U_{N}=X \backslash S_{N}$ for $N \geqslant 1 . T^{N}$ is well defined on $U_{N}$. Donote by $\beta^{N}=V^{N-1} T^{-k}(\beta)$ the family of all sets of the form $B_{0} \cap T^{-1} B_{1} \cap \ldots \cap T^{-(N-1)} B_{N-1}^{h_{i=0}}$, where $B_{0}, \ldots, B_{N-1} \in \beta$.

Ligmara 1. Tror every $f \in \mathrm{BV}$

$$
\operatorname{Var}(f \cdot g)=\sum_{B \in \beta} \operatorname{Var}_{B}(f \cdot g)
$$

T'roof. This is an easy consequence of the fact that $\beta$ is a partition and $\left.g\right|_{s}=0$.

Wo define $g_{N}$ by $\left.g_{N}\right|_{S_{N}}=0$ and $\left.g_{N}\right|_{\sigma_{N}}=g \cdot g \circ T \ldots g \circ T^{N-1}$. Wo will see that $T^{N}, U_{N}, S_{N}, g_{N}, \beta^{N}$ satisfy our conditions for $N \geqslant 1$. The only non-trivial thing to verify is $\operatorname{Var}_{N}<+\infty$. But this is a consequence of the following lemma:

Tismma 2. $\operatorname{Var} g_{N} \leqslant 2^{N-1}(\operatorname{Var} g)^{N}, N \geqslant 1$.

Proof. Gase $N=1$ is obvious. If it is tiue for some $N \geqslant 1$, then by Lemman 1:

T3ut

$$
\operatorname{Var} g_{N+1}=\operatorname{Var}\left(g_{N} \circ T \cdot g\right)=\sum_{\text {Disel }} \operatorname{Varr}_{g s}\left(g_{N} \circ T \cdot g\right)
$$

$$
\begin{aligned}
\operatorname{Var}_{H}\left(g_{N} \odot T \cdot g\right) & \leqslant\left\|g_{N}\right\|_{\infty} \cdot \operatorname{Var}_{I B} g+\operatorname{Var}_{B} g_{N} T \cdot\left\|g \cdot \chi_{B}\right\|_{\infty} \\
& \leqslant \operatorname{Var} g_{N} \operatorname{Var}_{B} g+\operatorname{Var}_{N} \operatorname{Var}_{B} g=2 \operatorname{Var} g_{B} \operatorname{Var}_{B} g .
\end{aligned}
$$

So Var $g_{N+1} \leqslant 2$ Vaxg $\operatorname{Varg}$. 
CoRoLlaRx 1. If we replace the hypothesis $\|g\|_{\infty}<1$ by $\left\|g_{N}\right\|_{\infty}<1$ for some $N \geqslant 1$, then $T^{N}$ satisfies our conditions.

Remark 3 . We will see later that

$$
\sup _{N} \operatorname{Var} g_{N}<+\infty \text {. }
$$

LEMma. 3. For every $B \in \beta^{N}, N \geqslant 1$,

$$
m(B) \leqslant\left\|g_{N}\right\|_{\infty} \leqslant\|g\|_{\infty}^{N} .
$$

CoRollaxx 2. Let $O$ be a closed interval and assume that for every $N \geqslant 1$ there exists $B \in \beta^{N}$ such that $B \supset C$. Then $m(O)=0$. So $m$ has no atoms. Moreover, collapsing all maximal closed intervals of measure 0 , we can assume that $\beta$ is a generator.

(We do it from now on.)

Lencma 4. For every $f \in \mathrm{BV}$,

$$
\sum_{B \in \beta} \operatorname{Var} P\left(f \cdot \chi_{B B}\right)=\operatorname{Var}(f \cdot g) .
$$

Proof. We notice that $\left.P\left(f \cdot \chi_{B}\right) \circ T\right|_{B}=f \cdot g \cdot \chi_{B}$. So wo havo $\operatorname{Var} P\left(f \cdot \chi_{B_{B}}\right)$ $=\operatorname{Var}_{B}\left(f \cdot g \cdot \chi_{B}\right)=\operatorname{Var}_{B}(f \cdot g)$ and we apply Lomma 1 .

LEMMA 5. Let a be a finite partition. Then

$$
\operatorname{Var}(f \cdot g) \leqslant \lambda \operatorname{Var} f+D \sum_{\lambda \in a}\left|\int_{\Lambda} f d m\right|
$$

where $D=\max _{A \in \alpha} \operatorname{Var}_{A} g / m(A)$ and $\lambda=\|g\|_{\infty}+\max _{A \in \alpha} \operatorname{Var}_{A} g$.

Proof. We have $\operatorname{Var}_{A}(f \cdot g) \leqslant \operatorname{Var}_{A} f \cdot\|g\|_{\infty}+\left\|f \cdot \chi_{A}\right\|_{\infty} \operatorname{Varr}_{A} g$ and $\left\|\cdot f \cdot \chi_{A}\right\|_{\infty}$ $\leqslant 1 / m(A)\left|\int_{A} f d m\right|+\operatorname{Var}_{A} f$. Since $\operatorname{Var}(f \cdot g)=\sum_{A \in a} \operatorname{Var}_{A}(f \cdot g)$, we get $(10)$.

LEMma 6. For every $\varepsilon>0$ there exists a partition a which is finite and

$$
\max _{A \in \alpha} \operatorname{Var}_{A} g \leqslant\|g\|_{\infty}+\varepsilon .
$$

Proof. Jumps of $g$ do not axeesd $\|g\|_{\infty}$. Thus, for (1Vory a $\in$ X and some interyal containing $x, \operatorname{Var}_{U_{x}} g<\|g\|_{\infty}+\varepsilon$. Taking a fincer than the cover $\left\{U_{\text {ansex }}\right.$ wo obtain (11).

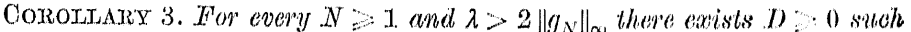
that for every $f \in \mathrm{BV}$ :

$$
\operatorname{Var} I^{N} f \leqslant \sum_{B \in \beta^{N}} \operatorname{Var} P^{N}\left(f \cdot \chi_{13}\right) \leqslant \lambda \operatorname{Var} f+D\|f\|_{1} .
$$

Proof. We notice that $P^{N} f=\sum_{B \in \beta} P^{N}\left(f \cdot \chi_{B}\right)$. We also have $\sum_{A \in \alpha}\left|\int f d m\right|$ $\leqslant\|f\|_{1}$. So Lemmas 4, 5 and 6 give Corollary 3 .

Since the sequence $\left(\left\|g_{N}\right\|_{\infty}\right)_{N=1}^{\infty}$ is submultiplicative, the limit lim $\left\|g_{N}\right\|_{\infty}^{1 / N}$ $=\vartheta$ exists.$$
N \rightarrow \infty
$$

PRoposirron 1. Given $x \in(\vartheta, 1)$ we can find $F \geqslant 0$ such that for every $f \in \mathrm{BV}$,

$$
\sum_{B \in \beta^{n}} \operatorname{Var} P^{n}\left(f \cdot \chi_{X 3}\right) \leqslant F\left(\|f\|_{1}+\varkappa^{n} \operatorname{Var} f\right)
$$

for $n=1,2, \ldots$

Iroof. First we fix $k, l \geqslant 1$. We will prove the following lemma:

Lwomm 7. Tet $\lambda_{k}, \lambda_{l}$ and $D_{l}, D_{l}$ be such that (12) holds with $N=\%$ and $N=l$, respectivety, if one replaces $\lambda$ by $\lambda_{7}, \lambda_{l}$ and $D$ by $D_{l}, D_{l}$, respectively. Then (12) holds with $N=k+l$ and $\lambda=\lambda_{k} \lambda_{l}$ and $D=D_{l_{k}}+\lambda_{k} D_{l}$.

Proof. We notice that for every $B_{1} \in \beta^{k}$ and $B_{2} \in \beta^{l}$.

$$
P^{k}\left(\chi_{B_{1}} \cdot P^{l}\left(\chi_{B_{2}} \cdot f\right)\right)=P^{k+l}\left(\chi_{B} \cdot f\right)
$$

whore $B=T^{-l} B_{1} \cap B_{2} \in \beta^{l+l}$. We use (12) for $P^{l}\left(f \cdot \chi_{B_{2}}\right)$ instead of $f$ :

$$
\sum_{J_{1} \in f^{\prime l c}} \operatorname{Var} P^{k+l}\left(\chi_{B_{2} \cap T^{\prime}-l_{I_{1}}} \cdot f\right) \leqslant \lambda_{k} \operatorname{Var} P^{l}\left(\chi_{I_{3}} \cdot f\right)+D_{k}\left\|P^{l}\left(\chi_{B_{2}} \cdot f\right)\right\|_{1}
$$

Wo sce that $\left\|P^{l}\left(\chi_{B_{2}} \cdot f\right)\right\|_{1} \leqslant \int_{J_{3}}|f| d m$. Sinee if $B_{1}$ runs through $\beta^{l}$ and $B_{2}$ runs through $\beta^{l}$, then $B_{2} \cap T^{-l} B_{1}$ runs through $\beta^{k+l}$, we have

$$
\begin{aligned}
\sum_{B \in \beta^{k+l}} \operatorname{Var} P^{k+l}\left(\chi_{B} \cdot f\right) & \leqslant \lambda_{k_{c}} \sum_{J_{2} \in \beta^{l}} \operatorname{Var} P^{l}\left(\chi_{B_{2}} \cdot f\right)+D_{k}\|f\|_{1} \\
& \leqslant \lambda_{l_{k}}\left(\lambda_{l} \operatorname{Var} f+D_{l}\|f\|_{1}\right)+D_{l k}\|f\|_{1} \\
& =\lambda_{l_{l}} \lambda_{l} \operatorname{Var} f+\left(D_{l_{l}}+\lambda_{l} D_{l}\right)\|f\|_{1} .
\end{aligned}
$$

So, we proved the lemma.

Now wo fix $M$ suce that $x>\left\|2 g_{M}\right\|_{\infty}^{1 / \lambda r}$. Wo prat $\lambda_{M}=x^{\pi T}$. Wo also fix some $\lambda_{0}$ and $\left.I\right)_{0}$ stuch that (12) holds with $\lambda=\lambda_{0}$ and $D=D_{0}$ for $N:-1,2, \ldots, M$. Wor any given $n$ wo an find $k, b$ such that $n=7 \cdot M C+l$

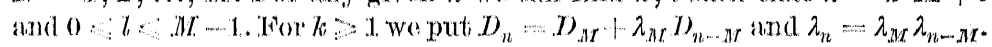
So, by induetion arement we see that for every $N \geqslant 1$ and $f \in \mathrm{BV}$ (12) holdes with $I)_{N}, \lambda_{N}$ instond of $D$ and $\lambda$. On the othere hand,

$$
\lambda_{n} \leqslant \lambda_{0} \lambda_{\lambda T}^{k}=\lambda_{0} / x^{l} \cdot x^{n}, \quad I_{n}=D_{0}\left(1+\lambda_{M}+\ldots+\lambda_{M T}^{k-1}\right) .
$$

Wo put $T=\max \left(D_{0} /\left(1-\lambda_{M}\right), \lambda_{0} / x^{M-1}\right)$. Tt is asy to see that (13) holds. 
CoRoldary 4. For every $N \geqslant 1$ and $f \in \mathrm{BV}$,

$$
\sum_{B \in \beta}\left\|P^{N}\left(f \cdot \chi_{B}\right)\right\| \leqslant\left(2 F^{\top}+1\right)\|f\| .
$$

Therefore, if $P$ is meant as an operator on $\mathrm{BV}$, then

$$
\sup _{N}\left\|P^{N}\right\| \leqslant 2 F+1 \text {. }
$$

Proof. For terms $P^{N}\left(f \cdot \chi_{B}\right)$ with the property

$$
\operatorname{Var} P^{N}\left(f \cdot \chi_{B}\right)>\left\|P^{N}\left(f \cdot \chi_{B}\right)\right\|_{1}
$$

we use (18). Other terms give at most $\|f\|_{1} \leqslant\|f\|$.

LEMCMA 8. For any $f \in \mathrm{BV}$ and a finite partition a,

$$
\operatorname{Var} E_{m}(f \mid \alpha) \leqslant \operatorname{Var} f,
$$

where $E_{m}(f \mid \alpha)$ is the conditional expectation of $f$ with respect to $a$.

Propostrion 2, There exist $N \geqslant 1$ and a finite-dimensional operator K on $\mathrm{BV}$ such that $\left\|P^{N}-K\right\|<1$.

Proof. We choose $N$ and a partition $\alpha$ such that Lemma 5 holds for $g_{N}$ with some $\lambda<1 / 2$. Let $E f=E_{m}(f \mid \alpha)$ for every $f \in \mathrm{BV}$ and $K=P^{N} E$. We will prove that this choice is good.

Let us fix $f \in \mathrm{BV}$ and take $h=f-t_{m}(f \mid \alpha)=(I-E) f$. For overy $A \in \alpha, \int_{A} h d m=0$, so $\operatorname{Var} P^{N} h \leqslant \lambda \operatorname{Var} h \leqslant 2 \lambda \operatorname{Var} f$ (Lemma 8) and $\left\|P^{N} h\right\|_{1}$ $\leqslant\|h\|_{I}^{A}=\sum_{B \in \beta} \int_{B}|h| d m$. We see that $\left\|h \cdot \chi_{B}\right\|_{\infty} \leqslant 2\left\|f \cdot \chi_{B}\right\|_{\infty} \leqslant 2 \operatorname{Var}_{B} f$ and $m(B) \leqslant\left\|g_{N}\right\|_{\infty} \leqslant \lambda$ for $B \in \beta^{N}$, so $\left\|P^{N} h\right\|_{1} \leqslant 2 \lambda\|f\|$. So $\left\|P^{N} h\right\| \leqslant 2 \lambda\|f\|$. Wo recall that $2 \lambda<1$. of $P$.

§ 2. In this section we give a description of some spectral properties

THEOREM 1. If $P$ is meant as operator on $\mathrm{BV}$, then:

(a) $\sigma(P) \cap S^{1}$ consists of a finite number of simple poles of the resolvent of $P$. Moreover, $\sigma(P) \cap S^{1}$ is a union of full cyctic groups.

(b) Other points of $\sigma(P)$ are contained within a circle of radius $r \in(0,1)$.

(c) If $\sigma(P) \cap S^{1}=\left\{\xi_{1}, \ldots, \xi_{L}\right\}$, we denote by $Q_{j}$ the projector on the corresponding eigenspace, $j=1, \ldots, L$; then $I$. admits the reprosentation

$$
P=\sum_{j=1}^{L} \xi_{j} Q_{j}+I R
$$

where $R: \mathrm{BV} \rightarrow \mathrm{BV}$ and $\varrho(R)=\inf _{N}\left\|R^{N}\right\|^{1 / N}<r$. Operators $Q_{1}, \ldots, Q_{L}, R$ commute and, moreover, $Q_{i} Q_{j}=0 \stackrel{N}{N}$ for $i \neq j$ and $Q_{i} R=R Q_{i}=0$ for $i, j$
$=1,2, \ldots, L$.
Proof. All these facts are implied by Proposition 2 and (19), by using the uniform orgodic theorem (see [3]), except for the possibility to represent $\sigma(P) \cap S^{1}$ as a union of full cyclic gxoups. It is a consequence of the theory of positive operators (see [4]). We would like to quote one theorem which refers to our situation.

Let $Y$ bo a Banach lattice. We assume that the absolute value on $X$ allows an extension on the complexification of $Y$. Let $U: Y \rightarrow Y$ be a positive operator such that there exists a strictily positive functional on $X$ which is an eigenvector of $U^{*}$ pertaining the cigenvalue $\varrho(U)$. Then the peripheral point spectrum of $U$ is cyclic $\left(\xi \in \sigma_{P}(U),|\xi|=\varrho(U)\right.$ $\left.\Rightarrow \xi(\xi /|\xi|)^{n} \in \sigma_{P}(U), n=1,2, \ldots\right)$.

Trmorem 2 . Operators $Q_{1}, \ldots, Q_{L}, R$ have unique extensions to operators on $I_{1}$. Moreover, $Q_{j}\left(L_{1}\right) \subset \mathrm{BV}$ and $Q_{j}$ is bounded as an operator from $I_{1}$ to $\mathrm{BV},\left\|Q_{j}\right\|_{1} \leqslant 1, j=1, \ldots, L$, and $\sup _{N}\left\|R^{N}\right\|_{1}<+\infty$. For every $f \in L_{1}$ $\lim _{S \rightarrow \infty} R^{N} f=0$.

Troof. Wo notice that for $j=1, \ldots, L$ and $\xi \in S^{1}$

$$
\lim _{N \rightarrow \infty} \frac{1}{N} \sum_{k=0}^{N-1}(P / \xi)^{k}=\left\{\begin{array}{lll}
0 & \text { as } & \xi \notin\left\{\xi_{1}, \ldots, \xi_{L}\right\} \\
Q_{j} & \text { as } \quad \xi=\xi_{j}
\end{array}\right.
$$

where the convergenes is in BV. But $\|P / \xi\|_{1}=1$, so $Q_{j}$ can be defined on $I_{1}$ by $(29)$, bccatuse $\mathrm{BV}$ is dense in $I_{1}$, and the extension is unique. Using Tomma 8 and Corollary 5 we easily prove other pointis.

Now wo are ready to analyse the situation when $\sigma(P) \cap S^{1}$ consists of 1 only. This allows us to describe the eigenvalues of $P$ in the general oase.

Tat $P=Q+R$ be the decomposition from Theorem 1 . By $f_{1} \wedge f_{2}$ we druote $\min \left(f_{1}, f_{2}\right)$. Wo write $T f$ instead of $f \circ T$.

THEorm. 3. There exist nonnegative functions $\varphi_{1}, \ldots, \varphi_{s} \in \mathrm{BV}$ and $\psi_{1}, \ldots, \psi_{s} \in I_{\infty}$ such that:

(a) Tror ever'y $f \in T_{1}$,

$$
Q f=\sum_{i=1}^{s} m\left(\psi_{i} \cdot f\right) \psi_{i}
$$

(b) $I^{\prime} p_{i}=p_{i}, x^{p_{i}}=p_{i}$ for $i=1, \ldots, s$.

(c) $m\left(p_{i} \psi_{j}\right)=\delta_{i j}, \quad \psi_{i} \wedge \psi_{j}=0=\phi_{i} \wedge \psi_{j}$ as $i \neq j ; \quad m\left(p_{i}\right)=1$ for $i=1, \ldots, s$.

(1) There exist measurable sets $C_{1}, \ldots, C_{s} \subset X$ such that $\psi_{i}=\chi_{C_{i}}$ a.e. for $i=1, \ldots, s$ and $X=\bigcup_{i=1}^{s} O_{i}$ a.e. 
(e) $\bigcap_{N=1}^{\infty} T^{N}\left(L_{1}\right)=\bigcap_{N=1}^{\infty} T^{N}\left(L_{\infty}\right)=\left\langle\psi_{\mathbf{1}}, \ldots, \psi_{s}\right\rangle$ (vector space spanned by $\left.\psi_{1}, \ldots, \psi_{s}\right)$.

(f) For every $f \in L_{1}, T^{N} f \rightarrow Q^{*} f$ in $\sigma\left(L_{1}, \mathrm{BV}\right)$-topology as $N \rightarrow+\infty$. Simitarly, for every $f \in I_{\infty}, T^{N} f \rightarrow Q^{*} f$ in $\sigma\left(L_{\infty}, L_{1}\right)$-topology and

$$
Q^{*} f=\sum_{i=1}^{s} m\left(p_{i} \cdot f\right) \psi_{i} .
$$

Proof. $Q$ is a positive operation by (21). Tet $Z=\mathscr{R}(Q)=\operatorname{ker}(I-P)$. Then $Z$ is a Banach sublattiee of $\mathrm{BV}$ and $L_{1}$. Indeed, let $f_{1}, f_{2} \in Z$. Them $Q\left(f_{1} \wedge f_{2}\right) \leqslant Q f_{1} \wedge Q f_{2}=f_{1} \wedge f_{2}$. But $Q$ preserves $m$ and we have $m\left(Q\left(f_{1} \wedge f_{2}\right)\right)$ $=m\left(f_{1} \wedge f_{2}\right)$. So $Q\left(f_{1} \wedge f_{2}\right)=f_{1} \wedge f_{2}$ a.e.

Let $\Delta=\{p \in Z: m(p)=1$ and $p \geqslant 0\}$. Wo notice that if $p, p^{\prime}$ are two distinct extreme points of $\Delta$, then $p \wedge p^{\prime}=0$. So extreme points of $\Delta$ are linearly independent and there is only a finite number of them. We denote them by $p_{1}, \ldots, p_{s}$. Hence $s \leqslant \operatorname{dim} Z$. On the other hand, $s \geqslant \operatorname{dim} Z$, since $p_{1}, \ldots, p_{s}$ span $\Delta$, by the Krein-Millman theorem, and $\Delta$ spans $Z$, because $\Delta=Q\left(\left\{f \in L_{1}: f \geqslant 0\right.\right.$ and $\left.\left.m(f)=1\right\}\right)$. So $s=\operatorname{dim} Z$. For every $f \in Z$,

$$
f=\sum_{i} \varphi_{i}^{*}(f) \varphi_{i},
$$

where $\left(p_{i}^{*}\right)_{i=1}^{s}$ is the basis dual to $\left(p_{i}\right)_{i=1}^{s}$. So for every $f \in L_{1}$,

$$
Q f=\sum_{i} p_{i}^{*}(Q f) \varphi_{i}=\sum_{i} \mu_{i}(f) \varphi_{i},
$$

where $\mu_{i}=Q^{*} p_{i}^{*}$ is a positive functional on $L_{1}$. As such, $\mu_{i}$ can be represented as

$$
\mu_{i}(f)=m\left(\psi_{i} f\right), \quad f \in L_{1},
$$

where $\psi_{1}, \ldots, \psi_{s} \in L_{\infty}$. Wo obtained (a). Wo notice that $P^{*}=T$. Tsing $P Q f=Q f=Q P f=\sum_{i} m\left(p_{i} P f\right) p_{i}$, we infer that $m\left(P^{*} \psi_{i} \cdot f\right)=m\left(\psi_{i} \cdot I \cdot f\right)$ $=m\left(\psi_{i} f\right)$ for cvery $f \stackrel{i}{\in} L_{1}$ and $i=1, \ldots, s$. So $P^{*}\left(\psi_{i}\right)=\psi_{i}=T^{\prime} \psi_{i}$.

Now we use $Q^{2}=Q$. We have $\sum_{i} m\left(\psi_{i} f\right) \varphi_{i}=Q f=Q^{2} f=Q\left(\sum_{i} m\left(\psi_{i} f\right) q_{i}\right)$ $=\sum_{i, j} m\left(\psi_{j} \varphi_{i}\right) m\left(\psi_{i} f\right) \varphi_{j}$. This gives $m\left(\psi_{i} \psi_{j}\right)=\delta_{i j}$. Now we checek (f). Iti is casy to see that $m(Q f \cdot h)=m\left(f \cdot Q^{*} h\right)=\sum_{i} m\left(\psi_{i} f\right) m\left(\psi_{i} h\right)$ and (if) follows by

$$
m\left(T^{N} f \cdot h\right)=m\left(f \cdot P^{N} h\right) \rightarrow m(f \cdot Q h)=m\left(Q^{*} \cdot f \cdot h\right) \quad \text { as } \quad N \rightarrow+1 \cdot \infty .
$$

We notice that $T^{N} \varphi_{i} \rightarrow \sum_{j} m\left(\varphi_{i} \varphi_{j}\right) \psi_{j}=m\left(\varphi_{i}^{2}\right) \psi_{i}$ in $\sigma\left(I_{\infty}, I_{1}\right)$-toljology as $N \rightarrow \infty, i=1, \ldots, s$. We also have (for $i \neq j$ ) $\psi_{i} \wedge T^{N} p_{j}=\left(\psi_{i} \wedge p_{j}\right) \circ T^{N}$
$=0, N=1,2, \ldots$ Wo get $0=m\left(p_{i} T^{N} p_{j}\right) \rightarrow m\left(\psi_{i} \psi_{j}\right) m\left(p_{j}^{2}\right)$ as $N \rightarrow \infty$. Since $m\left(p_{j}^{2}\right)>0, m\left(p_{i} \psi_{j}\right)=0$ for $i \neq j$. So, $\psi_{i} \wedge \psi_{j}=0$ for $i \neq j$

We completed (a), (b), (c) and (f).

Since $T 1=1$, so $Q^{*} 1=1$ and

$$
1=\sum_{i=1}^{s} \psi_{i}
$$

This gives (d).

Let $h \in \bigcap_{N=1}^{\infty} T^{N}\left(I_{\infty}\right)$. Wo choose a sequenee $h_{N} \in L_{\infty}$ such that $h=h_{N^{\circ}} \circ T^{N}$ and $\left\|h_{N}\right\|_{\infty} \leqslant\|h\|_{\infty}$. Since $\left(Q^{*} h_{N}\right)_{N=1}^{\infty}$ is bounded in $\left\langle\psi_{1}, \ldots, \psi_{8}\right\rangle$, wo can choosce a subsequence $N_{V_{c}} \rightarrow \infty$ such that $Q^{*} h_{N_{l_{c}} \rightarrow h_{0}}$ as $\eta_{i \rightarrow \infty}$. We will see that $h=h_{0}$ a.e. In fact, for every $f \in I_{1}$,

$$
\begin{aligned}
m(f \cdot h) & =m\left(f \cdot h_{N_{k_{k}}} \circ I^{N_{l_{k}}}\right)=m\left(P^{N_{k_{k}}} \cdot \cdot h_{N_{k_{k}}}\right) \\
& =m\left(\left(\mathcal{P}^{N_{l_{b}}}-Q\right) f \cdot h_{N_{k}}\right)+m\left(Q f \cdot h_{N_{l_{k}}}\right) .
\end{aligned}
$$

Since the first term tends to 0 and $m\left(Q f \cdot h_{N_{l}}\right)=m\left(f \cdot Q^{*} h_{N_{t}}\right)$, we obtain $m(f \cdot h)=m\left(f \cdot h_{0}\right)$ for every $f$. So, $h=h_{0}$ a.e. and $h \in\left\langle\psi_{1}, \ldots, \psi_{s}\right\rangle$. By approximation argument,

$$
\bigcap_{N=1}^{\infty} T^{N}\left(L_{1}\right)=\bigcap_{N=1}^{\infty} T^{N}\left(L_{\infty}\right)
$$

OoroxTARX 5. Xet $\mathscr{B}_{\infty}=\bigcap_{N=0}^{\infty} T^{-N}(\mathscr{B})$. Then $\mathscr{B}_{\infty}$ is generated by decomposition $\left(O_{1}, \ldots, O_{s}\right)$. The eigenspace of $T$ for the eigenvalue 1 is exactly $\left\langle p_{1}, \ldots, \psi_{s}\right\rangle$

$$
\text { Troof. } \bigcap_{N=1}^{\infty} T^{N}\left(I_{\infty}\right)=\left\{f \in L_{\infty}: f \text { is } \mathscr{B}_{\infty} \text {-measurable }\right\} .
$$

Remark 4. (a) $\psi_{1}, \ldots, \psi_{s}$ determine extreme rays of the positive cone in $\mathscr{R}^{*}\left(Q^{*}\right)$.

(b) Dynamiend systems $\left(T_{i}, v_{i}\right)$, where $T_{i}=\left.T\right|_{G_{i}}$ and $v_{i}=p_{i} \cdot m_{\text {, }}$ $i, 1, \ldots, s$, are exaset and $v_{i}$ is the only invariant measure for $T_{i}$ absolutely continuous with respecet to $\left.m\right|_{o_{i}}$.

TIINORIM 4. Thet $T$ be as in \$1. Fix some $M$ verifying $\sigma\left(P^{M}\right) \cap S^{1}=\{1$. Leet $p_{1}, \ldots, \varphi_{s}, \psi_{1}=\chi_{G_{1}}, \ldots, \psi_{s}=\chi_{G_{s}}$ be constructed by Theorem 3 apptied to $P^{\text {in }}$. Then there exists a permutation $\pi$ of the set $\{1, \ldots, s\}$ suoh that

(28) $\quad I p_{i}=p_{\pi(i)}, \quad T \psi_{\pi(i)}=\psi_{i}$ for $\quad i=1, \ldots, s$.

I tet $l_{1}, \ldots, l_{q}$ be the lengths of the independent cycles of $\pi$. Then $\sigma(P) \cap S^{1}$ is a union of cyclic groups of ranks $l_{x}, \ldots, l_{q}$. Let $l \in\left\{l, \ldots, l_{q}\right\}$ 
and $\xi^{l}=1$. The eigenspace for $\xi$ is generated by functions

$$
\frac{1}{l} \sum_{l=0}^{l-1} \xi^{-k} P^{k_{i}} p_{i}=\frac{1}{l} \sum_{k=0}^{l-1} \xi^{-k_{i}} p_{\pi^{k}(i)}
$$

where $i$ belongs to some cycle of length 7 . Similarly, the eigenfunctions for $T$ are given by

$$
\frac{1}{l} \sum_{l i=10}^{l-1} \xi^{k} \psi_{\pi^{l k}(i)}, \quad i=1, \ldots, s .
$$

Themark 5. It is casy to prove that

$$
s<\text { Card } a,
$$

where $\alpha$ is chosen as in Proposition 2.

\$ 3. We are going to discuss in detail the Bornoulli propertiy for the systems $\left(T_{i}, v_{i}\right)$ (see Romark $\left.4(b)\right)$. The restriotion to such dynamical systems is equivalent to the assumption that 1 is the only eigenvalue of $P$ on tho unit circle and there existis only one $p \in L_{1}, P_{p}=p$ and $m(\varphi)=1(\varphi \geqslant 0)$. The system $(T, \mu)$, where $\mu=p m$, is exact by $\$ 2$.

Let us introdue the following notation. Given partitions $\xi$, $\zeta$, wo defino

$$
\begin{aligned}
a(\xi, \zeta) & =\sum_{A \in E, B \in \zeta}|\mu(A \cap B)-\mu(A) \mu(B)| \\
& =\sum_{A \in \zeta} \sum_{B \in \zeta}|\mu(A \mid B)-\mu(A)| \mu(B) .
\end{aligned}
$$

By the Ornstein theory [5], the Bernoulli property is implied by

$$
\operatorname{sip}_{t, l \geqslant 1} d\left(\beta^{t}, \beta_{t+n}^{t+n+l}\right) \rightarrow 0 \quad \text { as } \quad n \rightarrow \infty \text {. }
$$

We denote by $\mathscr{\mathscr { H }}_{t_{2}}^{t_{2}}\left(0 \leqslant t_{1} \leqslant t_{2} \leqslant+\infty\right)$ the $\sigma$-algebra gencratied by tho partition $\beta_{l_{1}}^{t_{2}}=\bigvee_{t=t_{\mathrm{I}}}^{t_{2}} T^{-t}(\beta)$. Let;

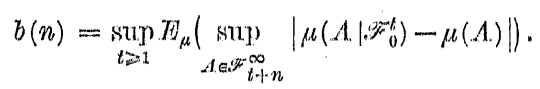

Let us notice that $2 b(n)$ is equal to the left-hand sicte of (33) (ef. [1]). So, we want to prove $b(n) \rightarrow 0$ as $n \rightarrow \infty$.

THEOREM 5. There exist $K \geqslant 0$ and $r \in(0,1)$ such that

$$
b(n) \leqslant \pi r^{n}, \quad n=1,2, \ldots
$$
Proof. Take $A \in \mathscr{F}_{t+n}^{\infty}, t, n \geqslant 1$. There exists $\tilde{A} \in \mathscr{F}_{0}^{\infty}=\mathscr{B}$ such
that $A=T^{-(t+n)} \tilde{A}$. Wo havo

$$
\mu\left(A \mid \mathscr{F}_{0}^{t}\right)=\frac{1}{\mu(B)} \int_{\mathcal{A}} \chi_{B} p d m=\frac{1}{\mu(B)} \int_{\tilde{A}} P^{t+n}\left(\chi_{B} \varphi\right) d m
$$

on $B \in \beta^{t}$. Of course, $\mu(A)=\mu(\tilde{A})=\int_{\tilde{A}} p d m$. This gives

This impliss

$$
\begin{aligned}
\left|\mu\left(A \mid \mathscr{F}_{0}^{t}\right)-\mu(A)\right| & \leqslant \int_{\tilde{A}}\left|P^{t+n}\left(\chi_{B} p\right) / \mu(B)-\varphi\right| d m \\
& \leqslant\left\|P^{t+n}\left(\chi_{B} p\right) / \mu(B)-\varphi\right\|_{1} .
\end{aligned}
$$

$$
b(n)<\sup _{l \rightarrow 1} \sum_{X_{\mathrm{C}} \beta}\left\|P^{l+n}\left(\chi_{I_{3}} p\right)-\mu(B) p\right\|_{1} \quad \text { for } \quad n \geqslant 1
$$

But

$$
\begin{aligned}
\left\|P^{t+1 \cdot n}\left(\chi_{B} p\right)-\mu(B) p\right\|_{1} & =\left\|P^{n}\left(P^{t}\left(\chi_{B} \varphi\right)\right)-\mu(B) \varphi\right\|_{1} \\
& \leqslant K_{1} \cdot r^{n}\left(\left\|P^{t}\left(\chi_{B} p\right)\right\|+\mu(B)\|\varphi\|\right)
\end{aligned}
$$

by Wheorem 1 (c) since in our case $\sigma(P) \cap \mathcal{S}^{1}=\{1\}$ and, by Theorem 2. the projection of $\mathcal{I}^{s t}\left(\chi_{1} q\right)$ on the eigenspace equals $m\left(P^{t}\left(\chi_{I} \varphi\right)\right) \varphi=\mu(B) \varphi$, Using Gorollary 4 we obtain

$$
b(n) \leqslant K_{1} \gamma^{n}\left(\sum_{B \in \beta^{\prime}}\left\|P^{t}\left(\chi_{B}^{*} \varphi\right)\right\|+\|\varphi\|\right) \leqslant K_{1}\left(2 F^{\prime}+2\right)\|\varphi\| r^{n} .
$$

So, wo cun pruti $\Pi=K_{1}\left(2 H^{2}+2\right)\|\varphi\|$.

ConotianT (6. The natural extension of the dynamical system $(T, v)$ is isomorphie with some Bernoulti shift.

(b) Ximit theorems hotd in the form proposed by Hofbaner and Keller in [1].

\$ 4. Miny axamples an bo found in [1]. Wo give ono examplo that eannotibe verified there.

Put $X:=[0,1]$ and leti $m$ bo the Lubesgate mosume on $[0,1]$. Let us

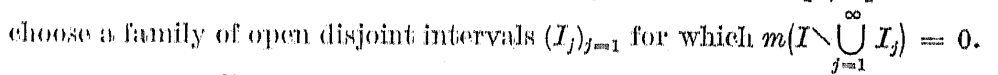

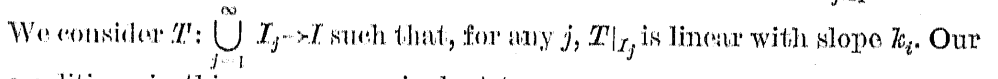
conditions in this cutse ane cquivalent to

$$
\inf _{1 \times i<+\infty} k_{i}>1, \quad \sum_{i=1}^{\infty} k_{i}^{-1}<+\infty .
$$


The following example shows that the second condition cannot bo omitted:

$$
I_{j}=\left(1 / 2^{j}, 1 / 2^{j-1}\right), \quad j=1,2, \ldots, \quad\left(T / I_{j}\right)(x)=2\left(x-1 / 2^{j}\right) .
$$

$T$ has no measures absolutely continuous with respect to $m$, because almost every point of $[0,1]$ tends to 0 under iterations of $\boldsymbol{T}$. This examplo shows that our method is very effective for such $T$.

I express my sincere thanks to M. Misiurewicz who read carcfully the first version of this paper and contributed many critical remarks and suggestions.

\section{References}

[1] F. Hofbauer, G. Keller, Ergodic properties of invariant measures for piecewise monotonic transformations, preprint.

[2] P. Walters, Invariant measures and equilibrium states for some mappings which extend distances, Trans. Amer. Math. Soc. 236 (1978), 121-153.

[3] N. Dunford, J. T. Schwartz, Linear operators, Part I. General theory, Wiloy, New York 1979.

[4] H. H. S chaefer, Banach lattices and positive operators, Springer-Vorlag, Berlin, Heidelberg, New York 1979.

[5] P. S. Ornstoin, Ergodic theory, randomness and dynamical systems, Yalo University Press, New Haven and London 1974. 